\section{Military Technical College Kobry El-Kobbah, Cairo, Egypt}

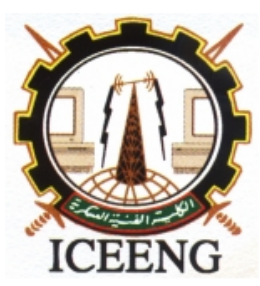

\author{
$8^{\text {th }}$ International Conference \\ on Electrical Engineering \\ ICEENG 2012
}

\title{
Narrowband Interference Cancellation in DS-CDMA System Using a Suppression Filter
}

\author{
By
}

A. Hanafy * Khairy El-Barbary ** Hossam Abou Bakr *** Ashraf M. Samy****

\section{$\underline{\text { Abstract: }}$}

In this paper the performance of the DS-CDMA system employing a suppression filter for rejection of narrow band interference (NBI) is provided. Two types of NBI, namely the binary phase shift keying (BPSK) and the single tone interferences, are considered. The behavior of the DS-CDMA system with and without a suppression filter over a Raleigh fading channel and under the effect of the two types of NBI is analyzed. The effects of suppression filter coefficients, DS-CDMA system parameters, and the NBI parameters on the performance of the system are evaluated analytically. It is shown that the suppression filter is more effective against the single tone NBI than in the case of the BPSK NBI. Moreover, it is proved that using a suppression filter with total three taps is sufficient to mitigate the effect of either the BPSK or single tone NBI.

\section{Keywords:}

CDMA; narrowband interference; suppression filter

* Egyptian Armed Forces

** Suez Canal University

*** Egyptian Armed Forces

**** Egyptian Armed Forces 


\section{Introduction:}

In recent years, code division multiple access (CDMA) has become one of the most used technology for wireless communication networks due to its high security, high spectral efficiency and its greater capacity. This multiple access method offers distinct advantages in suppressing interference and intentional jammers [1]. It is well known that the DSSS systems have inherent interference suppression capabilities. The spreading gain cannot provide sufficient degree of interference suppression capability due to the spreading gain is restricted and interfering signal is very strong. To overcome this problem, some signal processing techniques must be employed for further system performance improvement [2]. Under such circumstances, an effective NBI suppression scheme is required. NBI suppression before despreading in the receiver can improve the performance significantly. Many NBI suppression techniques in DSSS systems have been proposed in the previous researches and these are generally classified into three categories. These categories are time-domain methods, transform domain methods and multiuser detection (MUD) methods [3]. In [4] a linear suppression filter at a receiver of the single carrier DS-CDMA system is employed to reduce the NBI. In [5], an adaptive linear prediction version of least mean square (LMS) filter is applied to reject NBI. In [6], a non linear prediction technique is introduced and the dynamic convergence behavior of the adaptive nonlinear prediction filters is analyzed. In [7] a new kind of nonlinear filter called nonlinear Lattice and Transversal Joint (LTJ) filter is proposed. A discussion was introduced of their SNR improvement and convergence speed in NBI suppression in DS-SS communications and their complexity. For the transform-domain NBI suppression schemes, the received signals are firstly transformed into the frequency domain. After the NBI components are detected and notched out, the processed signals are inversely transformed into the time domain. The commonly used transforming schemes are Fourier transform, discrete cosine transform and Karhaunen-Loeve Transform (KLT) [8-10]. In [11], a new and powerful method for suppressing narrowband interference (NBI) in direct sequence spread spectrum (DS/CDMA) communication system is developed. This technique is based on the linear minimum mean square error (MMSE) algorithm for multiuser detection. The performance of the proposed method against NBI is compared with the performance of some previous linear and nonlinear NBI suppression methods. It is seen that this method outperforms all these previous techniques of NBI suppression. In [12], it is shown that the non-linear predictor does not provide the same performance as in CDMA systems as in single-user SS communication systems because the effectiveness decreases for the increasing number of users. In [13], linear predictor and adaptive NBI Re-estimation algorithms are combined with code-aided method. This proposed approach provides more performance improvement than the pure code aided approach. Compared with the transform-domain methods, the time domain filtering can eliminate the interference 
thoroughly with less impairment to useful signals. On the other side, time-domain filtering is of less complexity than the MUD scheme with more performance improvement [3].

In this paper, the average bit error probability of the DS-CDMA system subjected to BPSK and single tone NBIs and equipped with a suppression filter is derived. A well defined closed formula for the BER for each type of NBI is provided. The obtained results matches the results obtained in [4] for the case of BPSK NBI, moreover, this work is extended to cover the single tone NBI. Also, the comparison between the double sided and single sided filters in mitigating the effect of the two proposed NBIs is analyzed when the carrier of the NBI does not coincide with the carrier of the DSCDMA signal. The performance comparison of the system with and without a suppression filter under the effect of the two proposed types of NBIs is introduced. The behaviour of the suppression filter is evaluated for different situations, such as the effect of the number of taps and existence of an offset in frequency between the carrier of the NBI and the carrier of the DS-CDMA signal.

The paper is organized as follows. In Section 2, the system model is presented. In Section 3, the performance of the DS-CDMA system with the use of a suppression filter in presence of a BPSK NBI is derived. In Section 4, the performance of the DS-CDMA system with the use of a suppression filter in presence of a single tone NBI is derived. Section 5 provides the numerical results. The conclusions are provided in Section 6 .

\section{System model:}

The transmitted signal of the kth user in the DS-CDMA system can be expressed as [14] $\left.s_{k}(t)=\sqrt{2 P} b_{k}(t) c_{k}(t) \cos \left(2 \pi f t+\phi_{k}\right)\right]$

where $P$ is the transmitted power of the DS-CDMA signal, $b_{k}(t)$ is the baseband data sequence of user $\mathrm{k}, c_{k}(t)$ is the spreading waveforms of user $\mathrm{k}, f$ is the carrier frequency and $\phi_{k}$; is the initial phase of the carrier.

The binary source data sequence, $b_{k}(t)$ of the kth user is first spread by random binary sequence $c_{k}(t)$ with processing gain $N=T_{b} / T_{c}$, where $1 / T_{b}$ and $1 / T_{c}$ stands for the bit and chip rates, respectively. After that, the spread signal is modulated, with phase shift keying modulator (PSK), and then is transmitted.

The communication channel is considered as frequency nonselective Rayleigh fading channel with additive white Gaussian noise (AWGN). Moreover, the channel complex gain, $g_{k}$, is assumed to be with real and imaginary parts are assumed to be uncorrelated Gaussian distributed random variables, each with zero mean and variance $\sigma_{g}^{2}$, and is represented by $g_{k}=d_{k} \exp \left(j \psi_{k}\right)$. The amplitude $d_{k}$ has a Rayleigh distribution and the phase 
$\psi_{k}$ has a uniform distribution.

The receiver block diagram of the DS-CDMA system, with the use of a suppression filter, is shown in Fig. 1. The received signal enters the suppression filter, whose impulse response at a specific time is $\sum_{m=-M 1}^{M} \alpha_{m} \delta\left(t-m T_{c}\right)$, where $\left\{\alpha_{-M_{1}} \ldots \alpha_{0} \ldots \ldots \alpha_{M_{2}}\right\}$ are the tap weights with central tap $\alpha_{0}=1, M_{1} \geq 0$ and $M_{2} \geq 0$. It is noted that $M_{1}$ and $M_{2}$ stand for the numbers of taps on the left and right sides of the center tap, respectively. For $M_{1} \neq 0$ and $M_{2} \neq 0$, the filter is double sided suppression filter, whereas for $M_{1}=0$ and $M_{2} \neq 0$ or $M_{1} \neq 0$ and $M_{2}=0$, the filter is a single-sided suppression filter. The suppression filter uses the past and / or future values of the received signal to predict NBI current value, and then subtracts the predicted value from the received signal current value [15]. Assuming perfect channel estimation, the output of the suppression filter, $r_{k_{f}}$, is multiplied by the conjugate of the fading channel gain $g_{k}^{*}$ then passing through a DS despreader, and a PSK demodulator. Then, the output $x_{k}$ is compared to a zero threshold to decide the information content of the data bit of user $\mathrm{k}$.

\section{Performance of the DS-CDMA system in presence of a BPSK NBI:}

In this section, the theoretical performance of the DS-CDMA system with a suppression filter in presence of BPSK NBI interference is derived. The BPSK NBI is assumed to have a total power $J_{W}$. The BPSK NBI can be represented as

$$
J_{b}(t)=\sqrt{2 J_{W}} j(t) \cos (2 \pi(f+\Delta) t+\beta)
$$

Where $\Delta$ stands for the offset of the interference carrier frequency from the carrier frequency of the DS-CDMA signal, and $\beta$ denotes the interference phase of this interference subcarrier. The information sequence $j_{j}(t)$ has a bit rate $1 / T_{j}$, where $T_{j}$ denotes the duration of one bit. Therefore, the interference bandwidth is $B_{j}=2 T_{j}^{-1}$ (it is assumed that $B_{j}<B_{s}$ ). An important quantity is the ratio of the interference bandwidth to the spread spectrum system bandwidth $p=\frac{B_{j}}{B_{s}}=\frac{T_{c}}{T_{j}}$. Assuming perfect power control, the received signal associated with $\mathrm{K}$ asynchronous users in a Rayleigh fading channel can be expressed as

$$
r(t)=\sum_{k=1}^{K} \sqrt{2 P} b_{k}\left(t-\tau_{k}\right) g_{k} c_{k}\left(t-\tau_{k}\right) \cos \left(2 \pi f t+\varphi_{k}\right)+n(t)+J_{b}(t)
$$

where $\varphi_{k}=\phi_{k}-2 \pi f \tau_{k}$ is a random variable uniformly distributed in $[0,2 \pi), J_{b}(t)$ is a BPSK NBI, $n(t)$ is a sample function of a stationary zero mean complex Gaussian process, independent of signal and with power spectral density (psd) $N_{0} / 2$. Assume that 
the reference user is the k-th user and $\tau_{k}=0$ for notational convenience. The output of the suppression filter of the kth user, $r_{k_{f}}(t)$, can be expressed as

$\left.r_{k_{f}}(t)=\sum_{m=-M_{1}}^{M_{2}} \alpha_{m}\left\{\sqrt{2 P} b_{k}\left(t-\tau_{k}-m T_{c}\right) g_{k} c_{k}\left(t-\tau_{k}-m T_{c}\right) \operatorname{co}\left(2 \tau f\left(t-m T_{c}\right)+\varphi_{k}\right)+n\left(t-m T_{C}\right)+J_{b}\left(t-m T_{C}\right)\right\}\right\}$

Assuming that the internal interference term can be safely ignored [5], then the output of the correlator of the kth user corresponding to the zeroth data bit, $x_{k}^{b}$, can be expressed as

$x_{k}^{b}=D_{k}+\sum_{j=1 k \neq j}^{k} I_{k, j}+n_{k}+J_{k, b}$

where the terms on the right hand side of (5) are described below.

The first term, $D_{k}$, represents the desired signal of the reference user at the zeroth tap of the suppression filter that is given by

$D_{k}=\int_{0}^{T_{b}} \sqrt{2 P} b_{k}(t) g_{k} g_{k}^{*} d t=\sqrt{2 P} T_{b} b_{k}[0] g_{k} g_{k}^{*}=\sqrt{2 P} T_{b} b_{k}[0] d_{k}^{2}$

where $b_{k}[0]$ is the transmitted zeroth data bit. The useful signal power given $d_{k}$ is equal to $\left.S\right|_{d_{t}}=2 P T{ }_{b}^{2}\left(d_{k}^{2}\right)^{2}$

The second term $I_{k, j}$ is the multiple access interference (MAI) term due to the $\mathrm{j}^{\text {th }}$ user $(k \neq j)$ and it is given by [4]

$I_{k, j}=\sum_{m=-n_{1}}^{M} \alpha_{m}\left\{\sqrt{2 P} g_{j} g_{k} \cos \left(\theta_{j}\right)\left(b_{j}[-1] R_{k, j}(\tau(m))+b_{j}[0] \hat{R}_{k, j}(\tau(m))\right)\right\}$

where $\tau(m)=\tau_{j}+m T_{c}$ is uniformly distributed in $[0,2 \pi]$. In this term of (8) the variables $R_{k, j}(\tau)$ and $\hat{R}_{k, j}(\tau)$ are the partial cross-correlation functions defined by $R_{k, j}(\tau)=\int_{0}^{\tau} c_{j}(t-\tau) c_{k}(t) d t$ and $\hat{R}_{k, j}(\tau)=\int_{\tau}^{T_{k}} c_{j}(t-\tau) c_{k}(t) d t$ respectively. The variance of $I_{k, j}$ is given by [4]

$\sigma_{M A l}^{2}=\left(\frac{P T_{b}^{2}(K-1)\left(d_{k}^{2}\right)^{2}}{3 N}\right)\left[\sum_{m=-M_{1}}^{M} 2 \alpha_{m}^{2}+\sum_{m=-M_{1}}^{M} \alpha_{m} \alpha_{m+1}\right]$

The third term, $n_{k}$, is the channel noise component added to the kth user and it is given by

$n_{k}=\sum_{m=-M_{1}}^{M} \alpha_{m}\left\{\int_{0}^{T_{k}} n\left(t-m T_{c}\right) g_{k}{ }_{k} c_{k}(t) 2 \cos \left(2 \pi f t+\phi_{k}\right) d t\right\}$

From (10) the variance of the noise component, $n_{k}$, is given by [4]

$\sigma_{n}^{2}=\left(N_{0} T_{b} d_{k}^{2}\right) \sum_{m=-M_{1}}^{M} \alpha_{m}^{2}$

The fourth term, $J_{k, b}$, is the NBI component added to the kth user and it is given by 
$J_{k, b}=\sum_{m=-M_{1}}^{M_{2}} \alpha_{m}\left\{\int_{0}^{T_{b}} \sqrt{2 J_{W}} j\left(t-m T_{c}\right) g_{k}^{*} c_{k}(t) \cos \left(2 \pi \Delta\left(t-m T_{c}\right)+\eta\right) d t\right\}$

The variance of a BPSK NBI, $\sigma_{J, b}^{2}$, is deduced and it is given by

$\sigma_{J, b}^{2}=T_{b}^{2}\left(\frac{J_{W}}{N}\right) d_{k}^{2} \sum_{m_{1}=-M_{1} m_{2}=-M_{1}}^{M_{2}} \sum_{m_{1}}^{M_{2}} \alpha_{m_{2}} \sigma_{b}^{2}\left(m_{1}, m_{2}\right)$

Where $\sigma_{b}^{2}\left(m_{1}, m_{2}\right)$ is given by

$$
\sigma_{b}^{2}\left(m_{1}, m_{2}\right)=\int_{-1}^{1} \operatorname{sign}\left[\left(1-p \mid x-m_{1}+m_{2}\right)\right)(1-\mid x)\left(1-\frac{|x|}{N}\right) \cos \left(2 \pi q\left(x-m_{1}+m_{2}\right)\right) d x
$$

Where $\operatorname{sign}[x]=x$ or zero for $x \geq 0$ or $x<0$, respectively, and $q=\Delta T_{c}$ denotes the ratio of the offset of interference carrier frequency to the half spread spectrum bandwidth.

Collecting the above results, the decision variable $x_{k}^{b}$ given by (5), consists of a useful term $D_{k}$ and a total interference term [ MAI $\sum_{\substack{j \in k \\ j \neq k}}^{K} \mathbb{X}_{k \neq j}+$ Gaussian noise $n_{k}+$ narrowband interference $\left.J_{k, b}\right]$. The conditional variance of the total interference given, $d_{k}$, is represented as

$\sigma_{t k}^{2}=\left\{\left(\frac{P T_{b}^{2}(K-1) d_{k}^{2}}{3 N}\right)\left[\sum_{m=-M_{1}}^{M_{2}} 2 \alpha_{m}^{2}+\sum_{m=-M_{1}}^{M_{2}} \alpha_{m} \alpha_{m+1}\right]+\left(N_{0} T_{b} d_{k}^{2}\right) \sum_{m=-M_{1}}^{M_{2}} \alpha_{m}^{2}+\sum_{m_{1}=-M_{1}}^{M_{2}} \sum_{m_{2}=-M_{1}}^{M_{2}} \alpha_{m_{1}} \alpha_{m_{2}}\left[\frac{J_{W}}{N} T_{b}^{2} d_{k}^{2} \sigma_{b}^{2}\left(m_{1}, m_{2}\right)\right]\right\}$

When the number $(K)$ of active users is large and according to the central limit theorem given, $d_{k}$, the sum of the MAI term $\sum_{j=1, j \neq k}^{K} I_{k, j}$, and the narrowband interference term $J_{k, b}$ can be approximated by a conditional Gaussian random variable. Hence given $d_{k}$, all interference terms and the noise term can be combined into a single conditional Gaussian random variable. Therefore, the conditional signal to noise, MAI interference, and narrowband interference power ratio of the decision variable $x_{k}^{b}$ is given by $\gamma_{b}=\frac{\left.S\right|_{d_{k}}}{2 \sigma_{x_{k}^{b}}^{2}}$.

Then the conditional error probability, $P\left(e \mid\left\{d_{k}\right\}\right)$, can be written as

$P\left(e \mid\left\{d_{k}\right\}\right)=Q\left(\sqrt{2 \gamma_{b}}\right)=\int_{\sqrt{2 \gamma}}^{\infty} \frac{1}{\sqrt{2 \pi}} \exp \left(-\frac{z^{2}}{2}\right) d z$

where $\gamma_{b}$ has a chi-square distribution given by [15], $P_{\gamma, b}\left(\gamma_{b}\right)=\frac{1}{\bar{\Gamma}} \exp \left(-\gamma_{b} / \bar{\Gamma}_{b}\right)$ where $\bar{\Gamma}_{b}$ is the average signal to noise ratio and it can be written as $\left.\bar{\Gamma}_{b}=\left\{\left(\frac{(K-1)}{3 N}\right) \sum_{m=-M_{1}}^{M_{2}} 2 \alpha_{m}^{2}+\sum_{m=-M_{1}}^{M_{2}} \alpha_{m} \alpha_{m+1}\right]+\left(\frac{\overline{E_{b}}}{N_{0}}\right)^{-1} \sum_{m=-M_{1}}^{M_{2}} \alpha_{m}^{2}+2\left(\frac{\overline{E_{b}}}{N_{j}}\right)^{-1} \sum_{m=-M_{1} m_{2}=-M_{1}}^{M_{2}} \sum_{m_{1}}^{M_{2}} \alpha_{m_{2}} \alpha_{b}^{2}\left(m_{1}, m_{2}\right)\right\}^{-1}$

where $\overline{E_{b}}$ is the average energy per bit for the DS/CDMA system $\left(\overline{E_{b}}=P T_{b} E\left[d_{k}^{2}\right]\right), \bar{P}$ is the average power $\left(\bar{P}=P E\left[d_{k}^{2}\right]\right)$, and $N_{j}=J_{W} / W_{s s}$ represents the effective psd of the BPSK NBI in the total communication band. Recall that, our goal is to evaluate the 
performance of the system in terms of average BEP. For this purpose the conditional BEP in (14) has to be statistically averaged over the random parameter $\gamma$. The average $\mathrm{BEP}$ is given by

$$
P_{e_{a}, b}=\frac{1}{2}\left(1-\sqrt{\frac{\overline{\bar{\Gamma}_{b}}}{1+\bar{\Gamma}_{b}}}\right)
$$

\section{Performance of the DS-CDMA system in presence of a single tone NBI:}

In this section, the analytical performance of the DS-CDMA system with a suppression filter in presence of a single tone NBI is derived. The single tone NBI can be represented as

$J_{t}(t)=\sqrt{2 J_{W}} \cos (2 \pi(f+\Delta) t+\beta)$

The output of the correlator of the k-th user corresponding to the first data bit in presence of a single tone NBI , $x_{k}^{t}$, can be expressed in simplified form as in (5). It is clear that the first three terms ( the desired signal term, the MAI term and the noise component term) are identical for both types of NBIs, and they can be expressed by (6), (8) and (10) respectively, whereas the fourth single tone NBI term, $J_{k}^{t}$, is given by

$J_{k}^{t}=\sum_{m=-M_{1}}^{M_{2}} \alpha_{m}\left\{\int_{0}^{T_{k}} \sqrt{2 J_{w}} g_{k}^{*} c_{k}(t) \cos \left(2 \pi \Delta\left(t-m T_{c}\right)+\eta\right) d t\right\}$

The variance of the fourth term, $\sigma_{J, t}^{2}$, can be obtained by substituting of $p=0$ in (14) and it is given by

$\sigma_{J, t}^{2}=T_{b}^{2}\left(\frac{J_{W}}{N}\right) d_{k}^{2} \sum_{m_{1}=-M_{1}}^{M_{2}} \sum_{m_{2}=-M_{1}}^{M_{2}} \alpha_{m_{1}} \alpha_{m_{2}} \int_{-1}^{1}(1-|x|)\left(1-\frac{|x|}{N}\right) \cos \left(2 \pi q\left(x-m_{1}+m_{2}\right)\right) d x$

The average probability of error in the presence of the single tone NBI, $P_{e_{a v}}^{t}$, can be obtained by following the same analysis as in the previous section, and it is given by

$P_{e_{a r}, t}=\frac{1}{2}\left(1-\sqrt{\frac{\bar{\Gamma}_{t}}{1+\bar{\Gamma}_{t}}}\right)$

where $\bar{\Gamma}_{t}$ is the average signal to noise ratio in presence of the single tone NBI and it can be written as

$\overline{\Gamma_{t}}=\left\{\left(\frac{(K-1)}{3 N}\right)\left[\sum_{m=-M_{1}}^{M_{2}} 2 \alpha_{m}^{2}+\sum_{m \in-M_{1}}^{M_{2}} \alpha_{m+1} \alpha_{m+1}\right]+\left(\frac{\overline{E_{b}}}{N_{0}}\right)^{-1} \sum_{m=-M_{1}}^{M_{2}} \alpha_{m}^{2}+2\left(\frac{\overline{E_{b}}}{N_{j}}\right)^{-1} \sum_{m_{1}=-M_{1} m_{2}=-M_{1}}^{M_{2}} \sum_{m_{1}}^{M_{2}} \alpha_{m_{2}} \sigma_{t}^{2}\left(m_{1}, m_{2}\right)\right\}^{-1}$

\section{Numerical Results and Discussions:}

In this section, using the above analytical results, we provide some representative numerical curves illustrating the average bit error probability of the DS-CDMA system with a suppression filter in the presence of a BPSK NBI and single tone NBI. Unless stated otherwise, it is assumed that the ratio of the offset of the interference carrier 
frequencies to the half spread spectrum bandwidth, ${ }^{q}$, is equal to zero, that is, the carrier frequency of the DS-CDMA and the two proposed NBI are identical (i.e. $\Delta=0$ ).

Fig.2 shows the BER performance of the DS-CDMA system with a double-sided suppression filter and without a suppression filter as function of the signal-to noise ratio under the effect of a BPSK NBI or single tone NBI. It is important to mention that this results are obtained under assumption that there are 10 active users, the spreading code has 63 chips per data bit, the ratio $(\mathrm{P})$ of the interference $\mathrm{BW}$ to the spread spectrum $\mathrm{BW}$ in the case of the BPSK NBI equals 0.1, and the signal-to- interference power ratio is $-5 \mathrm{~dB}$. The two-sided filter has three taps and is symmetric (i.e., $M_{1}=M_{2}=M=1$ ). One can see that, without a suppression filter, the presence of either the BPSK NBI or the single tone NBI results in the same performance degradation. On the other hand, it is clear that employing a suppression filter enhances greatly the system performance, for both types of NBI. It is also shown that the suppression filter is more effective in suppressing the single tone NBI than the case of BPSK NBI.

Fig. 3 illustrates average probability of error of the system with a double-sided filter, single sided filter, and without a suppression filter as a function of $E_{b} / N_{j}$ in the presence of a BPSK NBI and a single tone NBI. It is shown that the average probability of error of the system without the suppression filter decreases greatly as ${ }^{E_{b}} / N_{j}$ increases, whereas the performance of the system with a double sided or single sided suppression filter is improved at small values of ${ }^{E_{b}} / N_{j}$ and this improvement is more significant in the presence of a single tone NBI. Moreover, it is shown that the performance of the system with a suppression filter under the effect of a single tone NBI does not affected by changing the value of $E_{b} / N_{j}$. It is also noted that at large values of $E_{b} / N_{j}$, for example at $E_{b} / N_{j}>20 d B$, the system with and without a suppression filter approximately have the same performance in the presence of any of the two proposed NBI. It is also shown that under the effect of a BPSK NBI the double sided filter outperforms the single sided filter at small values of $E_{b} / N_{j}$ and at the same number of taps, while the performance of the system under the effect of a single tone NBI is alike for both the double sided and single sided filters.

In Fig. 4 and Fig. 5, the BER of the DS-CDMA system with a double-sided suppression filter is plotted as a function of the number of active users $(K)$ and for different values of $E_{b} / N_{j}$ in the presence of a BPSK NBI and a single tone NBI respectively. It is seen that, for a given average BER and under the effect of any of the two proposed NBI, the system with a double sided suppression filter can support many more users than can the system without a suppression filter especially when $E_{b} / N_{j}$ is small. When the 
interference is not too large (i.e. $E_{b} / N_{j} \geq 20 \mathrm{~dB}$ ) the system employing a suppression filter can support almost the same number of users as can the system without a suppression filter.

Fig. 6 and Fig. 7 illustrate the BER performance of the system with a double-sided suppression filter as a function of the ratio $(q)$, at different values of the number of taps on each side and with $E_{b} / N_{j}=-5 \mathrm{~dB}$ and under the effect of a BPSK NBI and a single tone NBI respectively. In these figures It is seen that the performance of the system without a suppression filter $(M=0)$ is enhanced by increasing the ratio $(q)$, whereas the performance of the system with a suppression filter depends on the value of $(q)$ with different manners. It is seen that as the value of $q$ becomes very small, the average BER of the system with a suppression filter for $m \geq 1$ are almost identical. However, the performance improves as $M$ increases when $q$ increases. It is also shown that increasing the number of taps to be more than one tap in each side increases the complexity of the filter and does not add any performance gain to the system either for small or large values of the ratio $(q)$. On the other hand when $q$ is very large (i.e., $q \geq$ 0.85 in fig. 6 and $q \geq 0.9$ in fig. 7 ) the system with and without a suppression filter have the same performance.

In Fig, 8 and Fig. 9 the performance comparison of the DS-CDMA system with the use of double sided and single sided suppression filters and without a suppression filter is illustrated as a function of the ratio (q), total number of taps $=3$ and under the effect of a BPSK and a single tone NBIs respectively. It is shown that, the single sided suppression filter outperforms the double sided suppression filter at $\mathrm{q}=0.25$ and 0.75 under the effect of the two proposed NBIs, and this improvement in the performance is more significant in the case of a single tone NBI. It is also noted that the use of the double sided suppression filter is useless at $\mathrm{q}=0.25$ and 0.75 because the low-pass version of the autocorrelation of the interference is zero, so that the centre tap coefficient equals one and the other two tap coefficients equal zero.

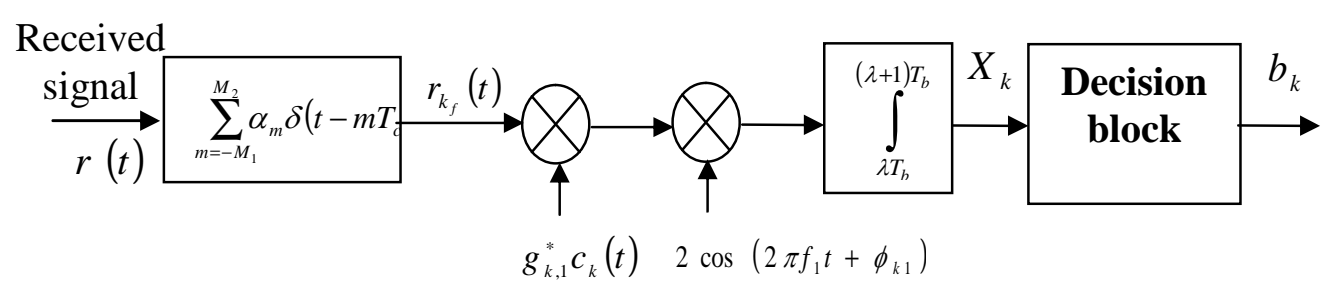

Figure (1):The receiver block diagram of DS-CDMA system with a suppression filter for the $k$-th user. 


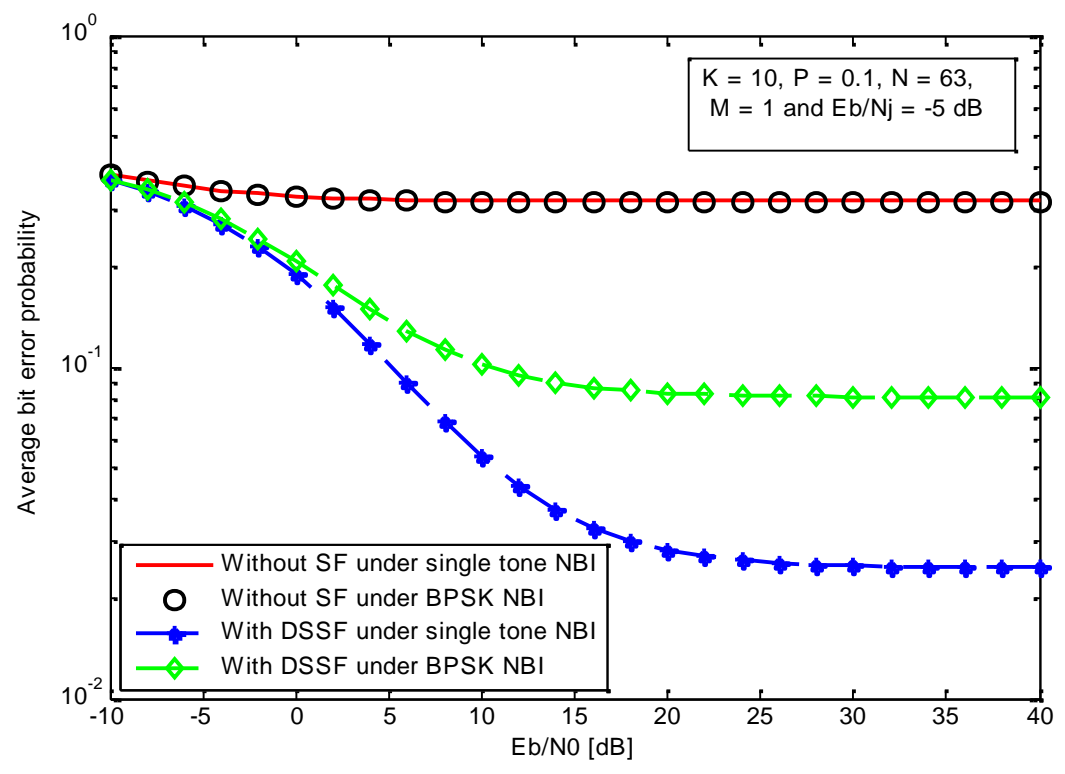

Figure (2):BER performance of DS-CDMA system with and without a suppression filter under BPSK and single tone NBIs.

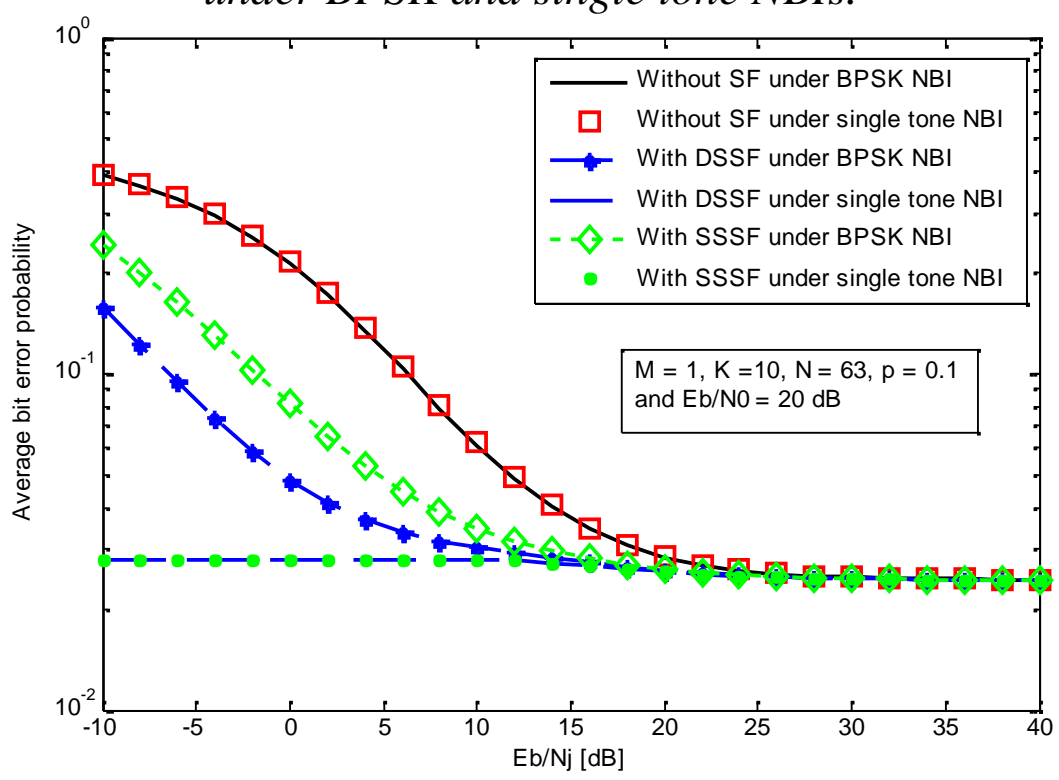

Figure (3):Performance comparison of DS-CDMA system under the effect of BPSK and single tone NB interferences with and without employing (DSSF) and (SSSF) as a function of $E_{b} / N_{j}$. 


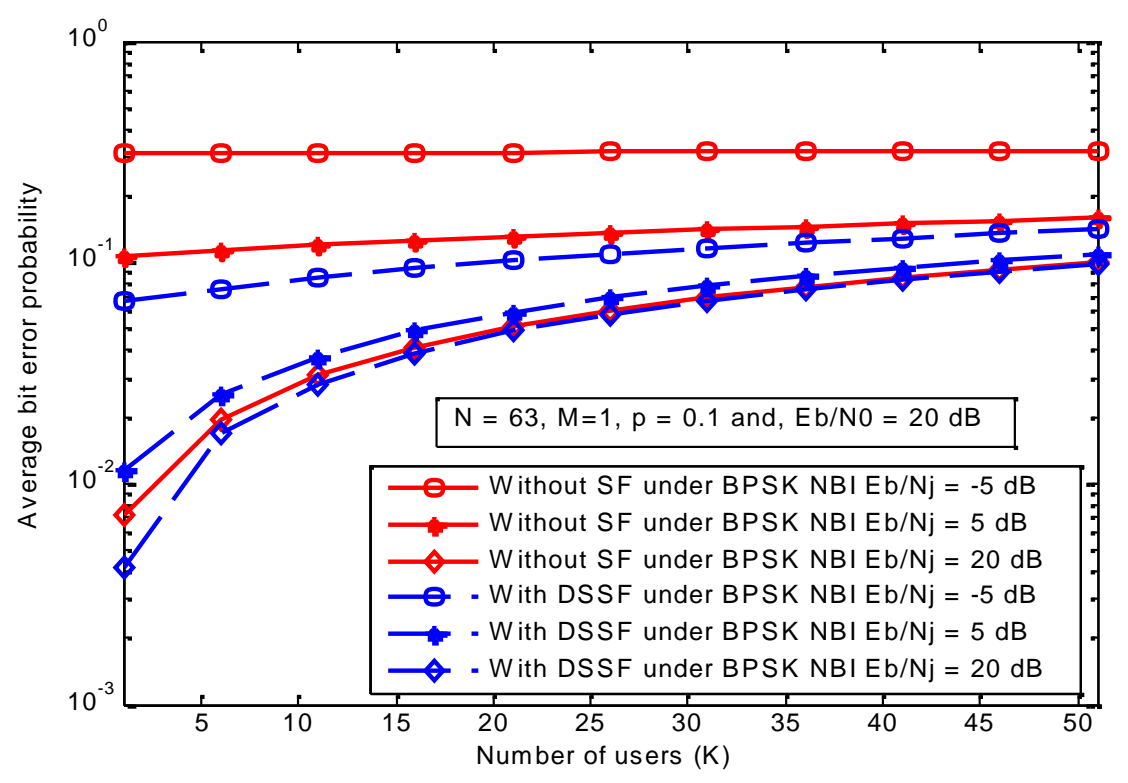

Figure (4):BER performance of DS-CDMA systems with and without suppression filter under the effect of BPSK NBI as a function of the number of active users $(K)$.

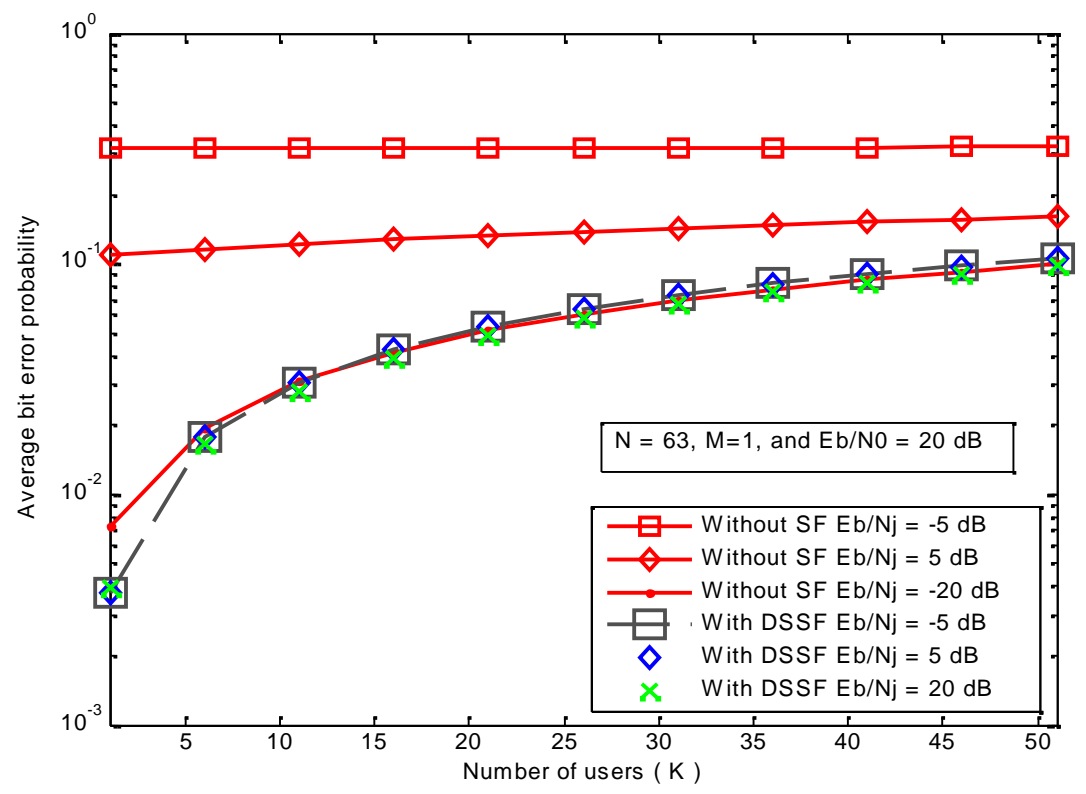

Figure (5):BER performance of DS-CDMA systems with and without suppression filter under the effect of single tone NBI as a function of the number of active users $(K)$. 


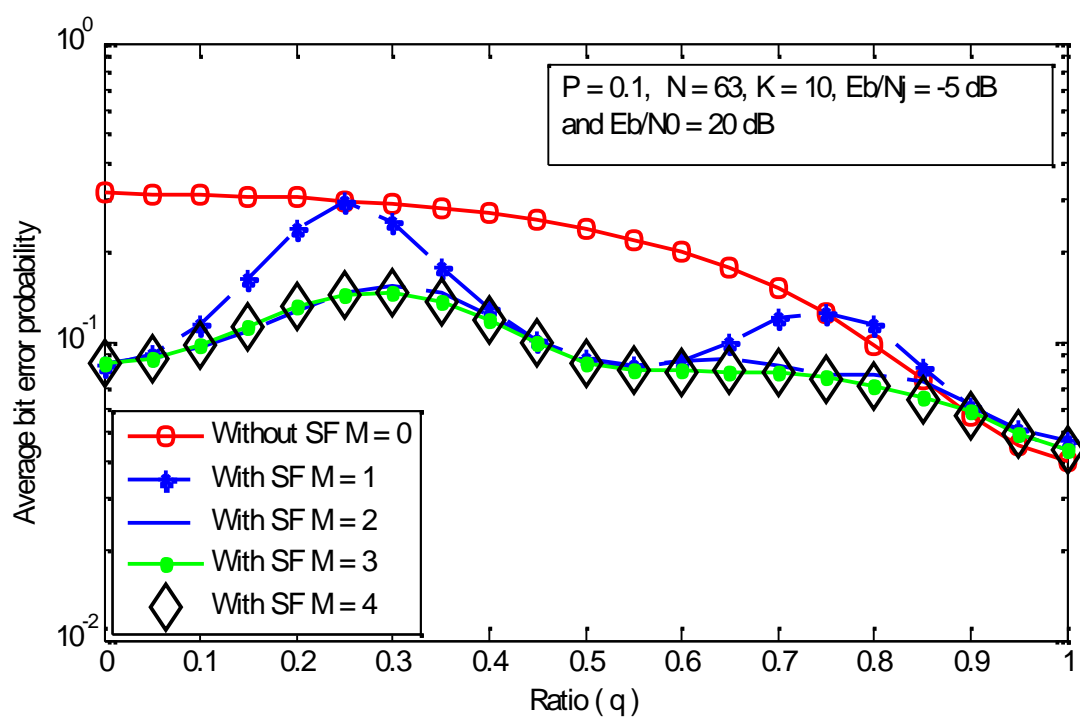

Figure (6):BER performance of DS-CDMA system under a BPSK NBI with and without a suppression filter as a function of the ratio $(q)$ and at different values of $M$.

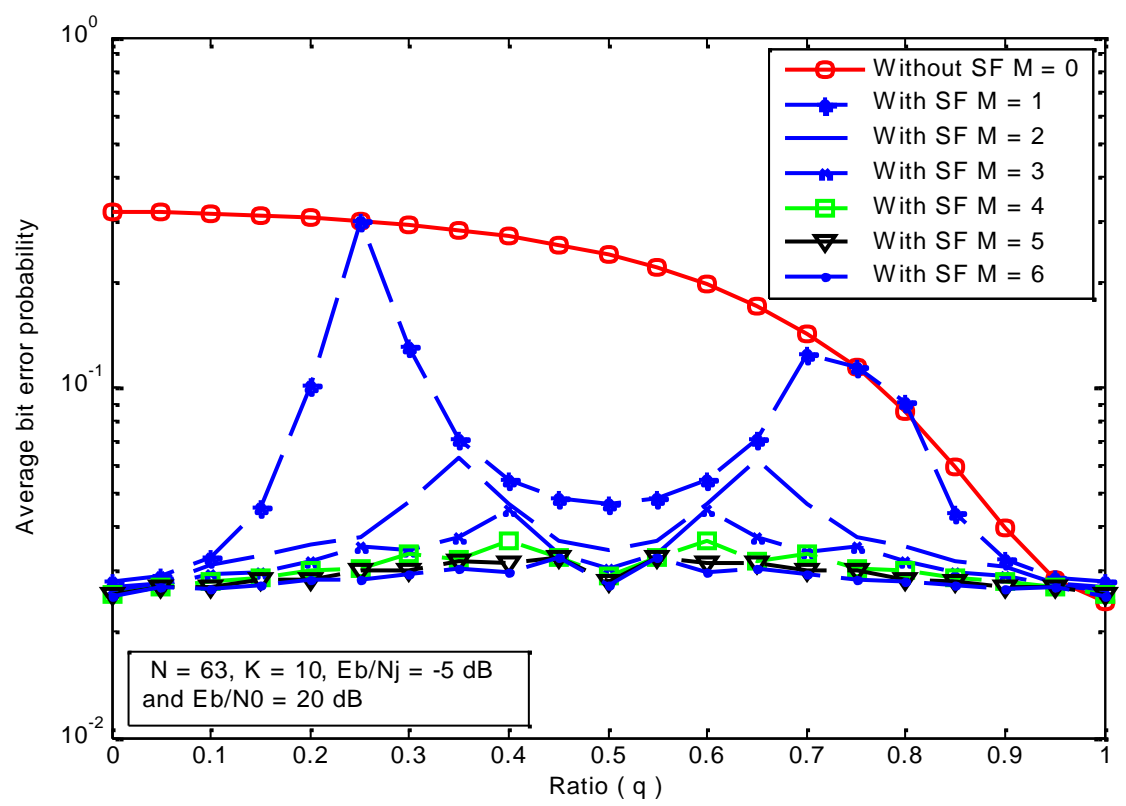

Figure (7):BER performance of DS-CDMA system under a single tone NBI with and without a suppression filter as a function of the ratio $(q)$ and at different values of $M$. 


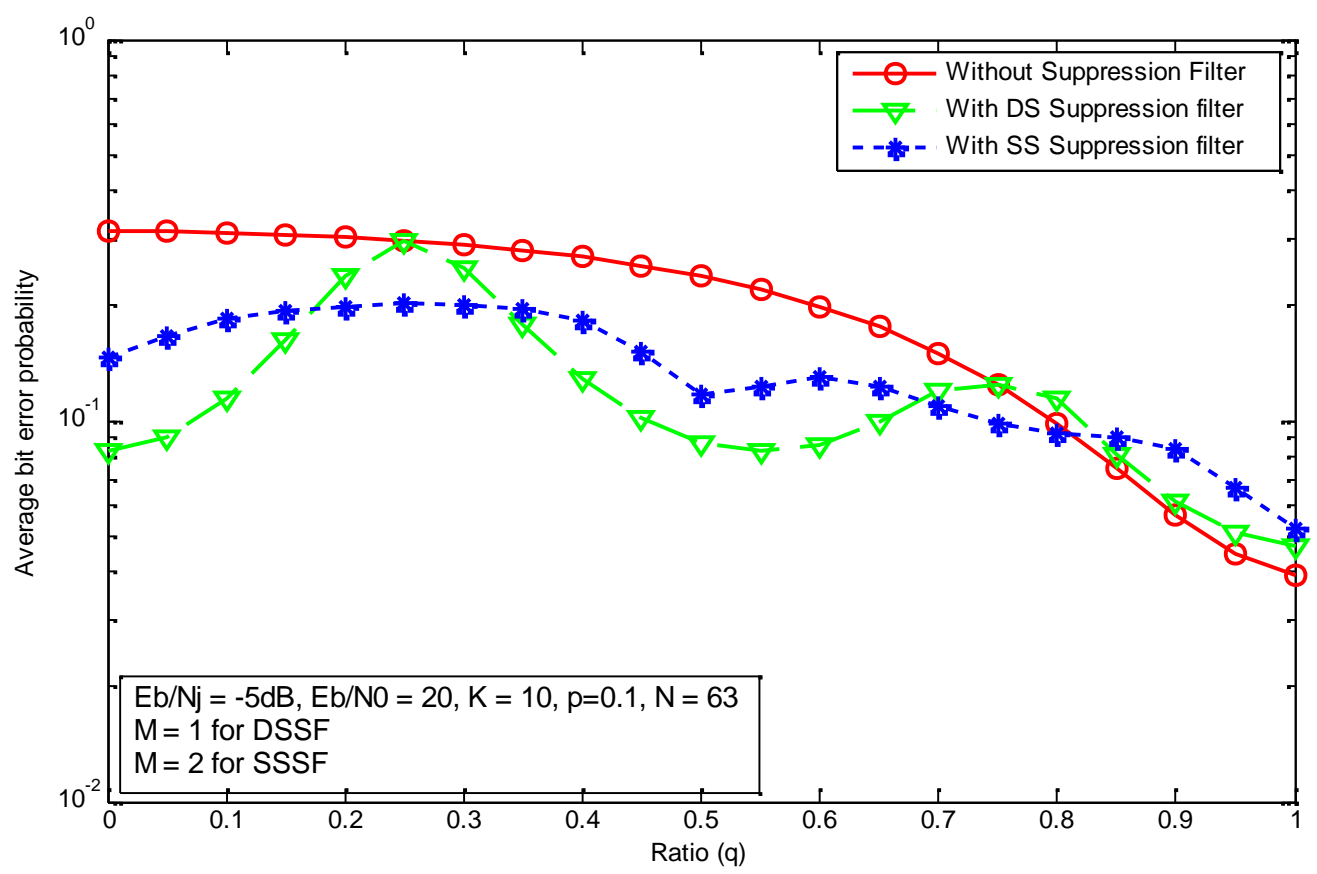

Figure (8):BER performance of DS-CDMA system under a BPSK NBI without and with employing (DSSF) or (SSSF) as a function of the ratio $(q)$.

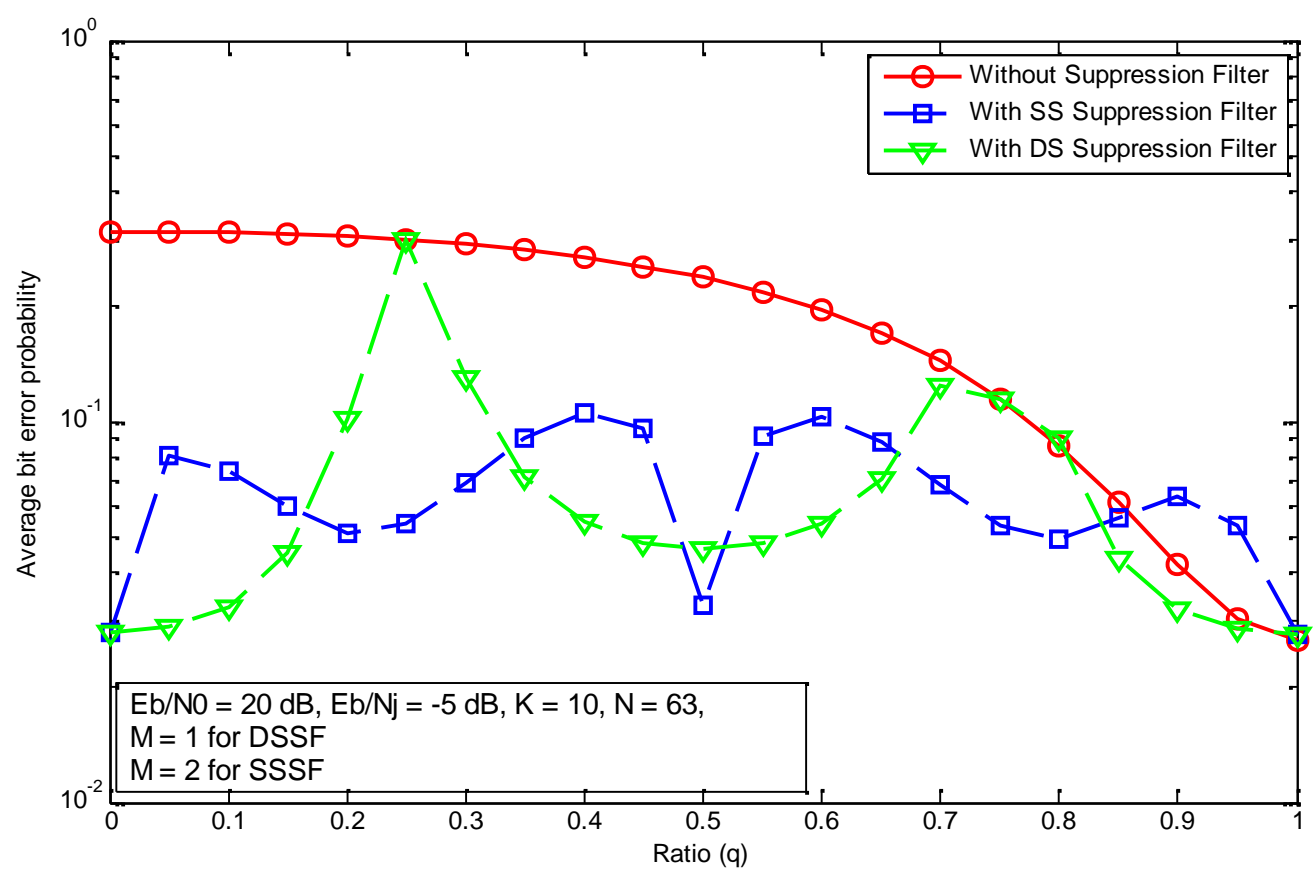

Figure (9):BER performance of DS-CDMA system under a singletone NBI without and with employing (DSSF) or (SSSF) as a function of the ratio $(q)$. 


\section{Conclusions:}

The BER performance has been derived analytically for a DS-CDMA system operating over a Rayleigh fading channel and employing a suppression filter and under the effect of a BPSK and a single tone NBIs. It has been shown that, the DS-CDMA system equipped with a suppression filter has a superior performance than the system without a suppression filter and consequently the system with a suppression filter can support more users. In addition, the double sided suppression filter outperforms the single sided suppression filter for the same number of total taps and in the presence of a BPSK NBI. Furthermore it has been investigated that, the performance of the system under the effect of the single tone NBI is similar for both the double sided and single sided filters. It has been shown that when the offset ratio $(q)$ equals to zero, the double sided suppression filter with three total taps is sufficient to mitigate the effect of the BPSK and the single tone NB interferences and increasing the number of total taps beyond three is not necessary.

\section{$\underline{\text { References: }}$}

[1] Kodithuwakku, J., Letzepis, N. and Grant, A., "Multi-User Decoder Assisted CodeAcquisition in CDMA Systems," Communications Theory Workshop (AusCTW), pp. $83-88,1-3$ Feb. 2011.

[2] Cheng Wang, Maode Ma, Rendong Ying, and Yuhang Yang, "Narrowband Interference Mitigation in DS-UWB Systems," IEEE Signal Processing, vol. 17, no. 5, May 2010.

[3] T. Zhao, Z. Yang and Y. Zhao "Partial-code-aided scheme for narrowband interference suppression in direct-sequence spread spectrum systems," IET Commun., 2010, Vol. 4, Iss. 18, pp. 2240-2251.

[4] J. Wang, "On the use of a suppression filter for CDMA overlay," IEEE Trans. Veh. Tech., vol. 48, pp. 405-414, March 1999.

[5] J Wang, and Milstein "Adaptive LMS Filters for Cellular CDMA Overlay Situations," IEEE Selected Areas in Commun., vol. 14, No. 8, pp. 1548-1559, October 1996.

[6] Fang Liu and Garth, L.M., 'Dynamic Convergence Analysis of Adaptive Nonlinear Filters for Narrowband Interference Rejection in Spread Spectrum Systems," International Symposium on Information Theory, pp. 1696 - 1700, 4-9 Sept. 2005.

[7] K. J. Wang and Y. Yao, "The nonlinear adaptive filters for narrow-band interference suppression in DS spread spectrum communications," Proc. IEEE conference in communication, circuits and systems 2006. 
[8] AZMI P., NASIRI-KENARI M.: 'Generalised Fourier transformdomain technique for narrowband interference suppression in CDMA communication systems', Electron. Lett., 2001, 37, (10), pp. 652-654

[9] AZMI P., NASIRI-KENARI M.: 'Narrow-band interference suppression in CDMA spread spectrum communication systems based on sub-optimum unitary transforms', IEICE Trans. Commun., 2002, E85-B, (1), pp. 239-246.

[10] P. Azmi and N. Tavakkoli: 'Narrow-Band Interference Suppression in CDMA spread spectrum communication systems using pre-processing based techniques in transform-domain,', Progress In Electromagnetics Research Letters, Vol. 3, 141$150,2008$.

[11] H. V. Poor, and X. Wang, "Code-Aided Interference Suppression for DS/CDMA Communications-Part I: Interference Suppression Capability," Proc. IEEE Trans. Commun., vol.45, no. 9, pp. 1101-1111, Sept. 1997.

[12] Yin Fulian and Guo Lili, "ZF Code-Aided Narrow-Band Interference Suppression for Synchronous DS-CDMA Systems," Wirless Commun, Networhing and Mobile Computing, International Conference, Oct. 2008.

[13] K. C. Ho, Xiaoning Lu, and Vandana Mehta, "Adaptive blind narrowband interference cancellation for multi-user detection," Proc. IEEE Trans. Commun., vol.60, no. 3, pp. 1024-1033, March 2007.D. Torrieri.

[14] Principles of Spread-Spectrum Communication Systems, Print Springer Science + Business Media, Inc, 2005.

[15] J. G. Proakis, Digital Signal Processing, Principles, Algorithms, and Applications, Person Prentice Hall, (Fourth Edition) 2007.

[16] M. K. Simon and M. S. Alouini, "Digital communication over fading channels: A unified approach to performance analysis, New York: Wiley, 2000. 